Article

\title{
Hybrid GA-SOCP Approach for Placement and Sizing of Distributed Generators in DC Networks
}

\author{
Oscar Danilo Montoya ${ }^{1,2, *(D)}$, Walter Gil-González ${ }^{3}$ (D) and Luis Fernando Grisales-Noreña ${ }^{3}$ (D) \\ 1 Facultad de Ingeniería, Universidad Distrital Francisco José de Caldas, Bogotá 11021, DC, Colombia \\ 2 Laboratorio Inteligente de Energía, Universidad Tecnológica de Bolívar, Cartagena 131001, Colombia \\ 3 Grupo GIIEN, Facultad de Ingeniería, Institución Universitaria Pascual Bravo, Campus Robledo, \\ Medellín 050036, Colombia; walter.gil@pascualbravo.edu.co (W.G.-G.); \\ luis.grisales@pascualbravo.edu.co (L.F.G.-N.) \\ * Correspondence: odmontoyag@udistrital.edu.co; Tel.: +57-3105461067
}

Received: 13 September 2020; Accepted: 26 October 2020; Published: 2 December 2020

check for updates

\begin{abstract}
This research addresses the problem of the optimal location and sizing distributed generators (DGs) in direct current (DC) distribution networks from the combinatorial optimization. It is proposed a master-slave optimization approach in order to solve the problems of placement and location of DGs, respectively. The master stage applies to the classical Chu \& Beasley genetic algorithm (GA), while the slave stage resolves a second-order cone programming reformulation of the optimal power flow problem for DC grids. This master-slave approach generates a hybrid optimization approach, named GA-SOCP. The main advantage of optimal dimensioning of DGs via SOCP is that this method makes part of the exact mathematical optimization that guarantees the possibility of finding the global optimal solution due to the solution space's convex structure, which is a clear improvement regarding classical metaheuristic optimization methodologies. Numerical comparisons with hybrid and exact optimization approaches reported in the literature demonstrate the proposed hybrid GA-SOCP approach's effectiveness and robustness to achieve the global optimal solution. Two test feeders compose of 21 and 69 nodes that can locate three distributed generators are considered. All of the computational validations have been carried out in the MATLAB software and the CVX tool for convex optimization.
\end{abstract}

Keywords: direct current networks; optimal power flow analysis; metaheuristic optimization; master-slave optimization; genetic algorithms; second-order cone programming

\section{Introduction}

Recently, the study and analysis of direct current (DC) networks have increased, due to the development of power electronics, advances in renewable generation, and the advantages over alternative current $(\mathrm{AC})$ networks [1,2]. Initially, the DC networks began to operate at high-level voltages in the power transmission for long-distance between systems, since it allows for reducing transmission power losses, enhancing grid stability systems, easily incorporating off-shore wind systems, and managing power flow both direction simply [3-5]. Consecutively, the integration of the DC networks has been widened to medium- and low-voltage level applications, generating concepts, such as the microgrids [6,7].

The main advantages of DC systems as compared to conventional AC systems can be summarized, as follows: (i) fewer power converter devices, (ii) no requirement of synchronizing generators, (iii) better voltage profiles, (iv) less power losses, and (v) do not require frequency and reactive power analysis and controls [8-10]. 
Two kinds of analysis are used to study DC networks. The first analysis is dynamic, being performed under the time domain, and integrates concepts as primary and secondary controllers [11,12]. The second analysis is static, which computes all of the state variables under stationary conditions. This analysis is known as tertiary control [13], which addresses power flow analysis [2,14], optimal power flow [15], economic dispatch strategies [16], and voltage stability analysis [17,18]. Furthermore, these analyses are integrated with other problems, such as the placement and sizing of multiple distributed generators (DGs) [19], as well as placement and operation of battery energy storage systems in DC networks [20].

In this study, we focus on the problem of the optimal placement and sizing of DGs in DC networks. Although, in the specialized literature, there are few approaches in this area. In [21], an analytical methodology for sizing energy storage systems in DC networks was proposed. This methodology evaluated the profiles of currents that were injected by energy storage systems in order to minimize power losses. In [22], a feasibility study for the integration of photovoltaic systems in a standalone building was carried out. This study considered strong weather scenarios for off-grid systems and variable electricity prices for the grid-connected system. In [23], a probabilistic optimization methodology for estimating uncertainties in loads, plug-in electric vehicles, and renewable generation in DC distribution networks was analyzed. In [19], a hybrid strategy for optimal location and dimensioning of DGs in DC grids was provided, which mixed a hyperplanes search method (HSM) with semidefinite programming (SDP) model. The HSM relaxed the binary variables, while the SDP computed the optimal power flow. In [24], a similar strategy using a sequential quadratic optimal power flow was presented. Both of the techniques ensure the optimal global solution only for a relaxed problem, where the complexity lies in the number of candidate solutions to recover the binary variables. In [25], a tutorial in the general algebraic modeling system (GAMS) for the optimal placement and dimensioning of DGs in DC grids were presented. In [26], the optimal placement and sizing of photovoltaic generators in DC networks were carried out, when considering an environmental-economic dispatch as an objective function. However, the approach presented in [26] was solved in GAMS, which does not guarantee the optimal global solution, since the problem is nonlinear non-convex mixed-integer. In [27], three metaheuristic methods for optimal location and dimensioning of DGs in DC systems were studied. A classical genetic algorithm was employed in the locations stage, which assigns the location of the DGs. While the black-hole, continuous genetic algorithm, and particle swarm metaheuristic methods were used to dimension the DGs. The main problem with these methods is that they have multiple tuning parameters that significantly affect their performance. Additionally, they do not ensure the optimal global solution to the problem. In [28], a methodology for optimal sizing of multiple devices, such as wind turbines, photovoltaic sources, and batteries in hybrid AC/DC grids was provided, which considered the life cycle cost and the life span of the renewable energy resources in the objective function.

After the revision mentioned above of the state-of-the-art, this research's main contributions can be summarized, as follows:

$\checkmark \quad$ The reformulation of the problem of the optimal placement and sizing of DGs in DC networks that allows transforming the original mixed-integer nonlinear programming (MINLP) model into a mixed-integer second-order cone programming (MI-SOCP) one.

$\checkmark \quad$ The solution of the proposed MI-SOCP model with a hybrid master-slave optimization methodology, where the master stage is guided by a genetic algorithm to determine the best locations for the DGs, while the slave stage allows for solving the optimal power flow problem by using the SOCP formulation via interior point methods to determine the best sizes of the DGs.

$\checkmark \quad$ The computational results demonstrate that the hybrid GA-SOCP approach has an efficiency higher than $70 \%$ to find the global optimal solution in both test feeders analyzed. In addition, the proposed approach presents better solutions than commercial solvers available in GAMS and classical metaheuristics such as genetic algorithms and particle swarm optimizer, and derived approaches 
It is worth mentioning that the master-slave hybrid GA-SOCP approach has not previously been proposed in specialized literature for addressing the problem of the optimal placement and sizing of DGs in electrical networks, even if these are under AC or DC paradigms of operation. This situation was identified as a gap in the scientific literature that this research tries to fill.

The remainder of this document is organized, as follows: Section 2 presents the original MINLP formulation of the problem of the optimal placement and dimensioning of DGs in DC networks by highlighting its nonlinearities and non-convexities. Section 3 presents the proposed hybrid optimization method, which is composed of a conic reformulation problem of the optimal power flow problem in DC networks and the classical GA. Section 4 exposes the main characteristics of the test feeders, which are composed of 21 and 69 nodes with radial structures, where the possibility of installing three DGs is considered. Section 5 presents all of the computational validations of the proposed hybrid GA-SOCP approach and their comparisons with nonlinear solvers available in GAMS and master-slave optimization approaches that are based on genetic algorithms and particle swarm optimization methods and its variants. Section 6 shows the concluding remarks derived from this work and some possible future researches in the area of distribution system optimization.

\section{MINLP Model}

The problem of the optimal placement and sizing of DGs in DC networks corresponds to an MINLP model since: (i) the problem of sizing of these power sources is modeled with binary variables, and (ii) the problem of the location of these DGs is modeled with continuous variables (power generation and voltage profiles). The optimization objective in this MINLP formulation corresponds to the minimization of the grid power losses produced by the current circulation in all the networks' branches. The mathematical structure of this objective function is presented in Equation (1).

$$
\min p_{\text {loss }}=\sum_{i \in \mathcal{N}} \sum_{j \in \mathcal{N}} G_{i j} v_{i} v_{j}
$$

where $p_{\text {loss }}$ is the value of the objective function regarding power losses in all of the branches of the network, $v_{i}$ and $v_{j}$ are the voltage values at nodes $i$ and $j$, respectively, and $G_{i j}$ is the conductance value taken from the admittance nodal matrix that defines the physical interconnection among all the nodes of the DC network. Observe that $\mathcal{N}$ is the set that contains all of the nodes of the network.

Regarding model constraints, these are related with operational conditions of the DC distribution networks. These constraints are presented below:

$$
\begin{array}{r}
p_{i}^{s}+p_{i}^{g d}-p_{i}^{d}=\sum_{j \in \mathcal{N}} G_{i j} v_{i} v_{j}, \forall i \in \mathcal{N} \\
p_{i j}=g_{i j} v_{i}\left(v_{i}-v_{j}\right), \forall(i, j) \in \mathcal{L} \\
\sum_{i \in \mathcal{N}} p_{i}^{g d} \leq \alpha \sum_{i \in \mathcal{N}} p_{i}^{d}, \\
\sum_{i \in \mathcal{N}} y_{i} \leq N_{d g}^{a v a}, \\
y_{i} p^{g d, \min } \leq p_{i}^{g d} \leq y_{i} p^{g d, \max } \forall i \in \mathcal{N} \\
p_{i j}^{\min } \leq p_{i j} \leq p_{i j}^{\max } \forall(i, j) \in \mathcal{L} \\
v^{\min } \leq v_{i} \leq v^{\max } \forall i \in \mathcal{N} \\
y_{i} \in\{0,1\} \forall i \in \mathcal{N}
\end{array}
$$

where $p_{i}^{s} p_{i}^{g d}, p_{i}^{d}$, and $p_{i j}$ represent the power generation in the voltage controlled source (slack nodes), power generation in distributed sources, power consumption in demand nodes, and power flow in distribution lines, respectively. $\alpha$ is a parameter that defines the percentage of power penetration of renewable generation as function of the total demand; $y_{i}$ is the binary variable associate with the 
location $\left(y_{i}=1\right)$ or no $\left(y_{i}=0\right)$ of a distributed generator at node $i$; $N_{d g}^{a v a}$ is the maximum number of distributed generators available for location in the DC network, $p^{g d, \min }$ and $p^{g d, \max }$ are the minimum and maximum power generation capabilities allowed for each distributed generator connected at node i. $p_{i j}^{\min }$ and $p_{i j}^{\max }$ are the lower and upper bounds for the power flow in line that connects nodes $i$ and $j$, respectively; and, $v^{\min }$ and $v^{\max }$ are the minimum and maximum voltage bounds in all nodes of the DC distribution network, respectively.

The complete interpretation of the MINLP model defined from (1) to (9) is as follows: Equation (1) defines the objective function of the problem regarding the minimization of the total grid power losses in the whole DC network; Equation (2) is the set of power balance equations applied at each node of the network; Equation (3) defines the amount of power that flows at each line of the DC grid. Expression (4) determines the maximum penetration of the distributed generation in the whole DC distribution network; Inequality constraint (5) bounds the number of generators available for location in the DC grid; inequalities (6), (7), and (8) present the upper and lower bounds for distributed generators, power flow in branches, and voltage variables, respectively. Finally, Expression (9) shows the binary nature of the decision variable regarding the location of distributed generators.

Remark 1. In the problem of the optimal placement and sizing of distributed generators in DC distribution networks is complex due to two main reasons: (i) the binary nature of the location problem makes necessary integer programming methods to define the best possible location of the distributed generators (master optimization problem); and (ii) the optimal sizing of the distributed generators for each possible location provided by the master stage is a nonlinear non-convex optimization problem due to the product between voltage variables in Expressions (1) to (3).

To solve the MINLP model (1)-(9), this research proposes a hybrid optimization approach based on genetic algorithms to address the problem of the optimal location of distributed generators and the second-order cone programming model to solve the problem of the optimal sizing of these devices. The main advantage of using SCOP instead of metaheuristics for optimal power flow analysis is that for each possible combination of distributed generator locations, the solution is the global optima [29], which is not possible with any particle swarm derived method that corresponds to the most classical approach for solving this kind of problems in literature [27].

\section{Proposed Hybrid GA-SOCP Optimization Approach}

We proposed a hybrid GA-SOCP approach, which works by using a master-slave optimization strategy, where the master stage guides the discrete part of the problem (i.e., defines the location of the DGs), and the slave stage deals with their optimal sizing, in order to address the problem of the optimal location of distributed generators in DC networks in this research.

\subsection{Slave Stage: Second-Order Cone Programming}

The SOCP is a branch of the convex optimization that allows transforming some nonlinear non-convex optimization problems into conic convex equivalents that guarantee the global optimum of the equivalent model [30]. In the case of the optimal power flow problem, the SOCP equivalent model has the same global optimum that the original nonlinear model, which is the main advantage of this technique in power system analysis [29]. Here, we present the SOCP version of the optimal power flow problem that addresses distributed generators' optimal sizing. For this purpose, let us suppose that all of the binary variables regarding the distributed generators' location, i.e., $y_{i}$ has been fixed by the master stage, which will be presented in this study. In this sense, the SOCP deals with the Equality constraints (2) and (3), which are the only continuous nonlinear non-convex constraints. 
The convexification of the optimal power flow problem is made using the following auxiliary variable:

$$
z_{i j}=v_{i} v_{j}
$$

note that, from (10), we know that $z_{i i}=v_{i}^{2}$ and $z_{j j}=v_{j}$, which implies that if we elevate both sides of (10) at the square, then we have:

$$
z_{i j}^{2}=z_{i i} z_{j j}
$$

Now, in order to obtain an equivalent representation of (11), we use the hyperbolic relation of the product between two variables as follows:

$$
\begin{aligned}
z_{i j}^{2} & =z_{i i} z_{j j}, \\
& =\frac{1}{4}\left(z_{i i}+z_{j j}\right)^{2}-\frac{1}{4}\left(z_{i i}-z_{j j}\right)^{2}, \\
\left(2 z_{i j}\right)^{2}+\left(z_{i i}-z_{j j}\right)^{2} & =\left(z_{i i}+z_{j j}\right)^{2}, \\
\left\|\begin{array}{c}
2 z_{i j} \\
z_{i i}-z_{j j}
\end{array}\right\| & =z_{i i}+z_{j j} .
\end{aligned}
$$

Note that Equation (12) is the conic equivalent of (11), since, to reach this result, only algebraic manipulations have been made; in addition, it is still non-convex due to the equality symbol; nevertheless, it can be relaxed with a lower-equal symbol as recommended in [13,31,32], which becomes (12) into a conic convex constraint, as presented below:

$$
\left\|\begin{array}{c}
2 z_{i j} \\
z_{i i}-z_{j j}
\end{array}\right\| \leq z_{i i}+z_{j j} .
$$

Remark 2. To obtain a SOCP equivalent of the optimal power flow problem, we can recur to substitute (11) into Expressions (1) to (3), which produce equivalent mixed-integer second-order cone programming (i.e., MISOCP) model to address the problem of the optimal placement and sizing of distributed generators in DC distribution networks.

The complete MISOCP model is presented below:

$$
\begin{gathered}
\min p_{\text {loss }}=\sum_{i \in \mathcal{N}} \sum_{j \in \mathcal{N}} G_{i j} z_{i j}, \\
p_{i}^{\varsigma}+p_{i}^{g d}-p_{i}^{d}=\sum_{j \in \mathcal{N}} G_{i j} z_{i j}, \forall i \in \mathcal{N} \\
p_{i j}=g_{i j}\left(z_{i i}-z_{i j}\right), \forall(i, j) \in \mathcal{L} \\
\left\|\begin{array}{c}
2 z_{i j} \\
z_{i i}-z_{j j}
\end{array}\right\| \leq z_{i i}+z_{j j}, \forall(i, j) \in \mathcal{N}
\end{gathered}
$$

$$
\text { Expressions (4) to (9), }
$$

Remark 3. Observe that the slave optimization stage solves the proposed MI-SOCP model once all the binary variables have been assigned on the master stage with the main advantage that the SOCP structure of the power flow model guarantees the global optimum finding [30]. 


\subsection{Master Stage: Genetic Algorithm}

The genetic algorithm is one of the most classical optimization methodologies from the family of combinatorial nature-inspired optimization methods [33]. The GA algorithm deals with complex optimization problems that involve integer (binary) variables, using three evolution rules: selection, recombination, and mutation [34]. Here, we select the GA optimizer to deal with the problem of the location of the distributed sources in DC networks using an integer combination that allows for minimizing the infeasibilities caused by classical binary codifications as recommended in [34]. The complete optimization structure of the proposed GA is presented below.

\subsubsection{Initial Population}

The most of nature-inspired optimization algorithms work with a list of potential solutions named "population". This list has the complete information regarding the solution of the problem under study. Here, we consider an integer population to determine the location of the DGs in the Dc network. This population $W^{t}$ (being $t$ the iterative counter) takes the following form:

$$
W^{t}=\left[\begin{array}{cccc}
w_{11} & w_{12} & \cdots & w_{1 a} \\
w_{21} & w_{22} & \cdots & w_{2 a} \\
\vdots & \vdots & \ddots & \vdots \\
w_{b 1} & w_{b 2} & \cdots & w_{b a}
\end{array}\right]
$$

where $a$ is the number of variables of interest and $b$ is the number of the individuals in the population.

Note that the component $w_{k m}$ in the initial population matrix is calculated, as follows:

$$
w_{k m}=\operatorname{round}(1+\operatorname{rand}(\cdot(n-1)), \forall k=1,2, \ldots, b, \forall m=1,2, \ldots, n .
$$

where round $(\cdot)$ determines the integer part of the argument and $\operatorname{rand}(\cdot)$ is a random number generated with a normal Gaussian distribution in the interval $[0,1]$. It is worth remembering that $n$ is the number of nodes and it corresponds to the cardinality of the set $\mathcal{N}$, i.e., $n=|\mathcal{N}|$.

Remark 4. An essential stage during the creation of the initial population is that it is mandatory to guarantee that each potential individual $W_{i}^{t}$ is different from each other $W_{j}^{t} ; \forall j \neq i$. This condition is known in genetic algorithms terminology as the diversity verification.

\subsubsection{Fitness Function Evaluation}

It is needed to define the fitness function in order to evaluate the initial population. The fitness function in metaheuristic optimization is the "adaptation" of the objective function to deal with possible infeasibilities in the solution space [35,36]. However, in the proposed MI-SOCP model, we guarantee that each individual is feasible, since the SOCP model is correctly constrained by the optimization model (14)-(18). In this sense, our proposed methodology's fitness function is equal to the objective function that is defined in Equation (14).

\subsubsection{Selection}

The selection (tournament) is the procedure where a subset of parents is chosen from the initial population to obtain a set of potential solutions (offsprings). Here, we select the most straightforward selection method that consists of selecting two individuals (a different one to each other) from the population, named as the parents. 


\subsubsection{Recombination}

The recombination is a heuristic procedure where the genetic information of the parents is crossed to generate offsprings. To present this procedure, let us consider two parents that are provided by the selection stage as follows:

$$
\begin{aligned}
& W_{i}^{t}=\left[\begin{array}{lllllllll}
1 & 0 & 1 & 1 & 0 & 1 & 1 & 0 & 0
\end{array}\right] \\
& W_{j}^{t}=\left[\begin{array}{llllllllll}
0 & 0 & 1 & 0 & 0 & 0 & 1 & 0 & 1 & 1
\end{array}\right] \text {. }
\end{aligned}
$$

Note that, in this example, the each parent has 10 genes $(n=10)$. To make the recombination it is generate a random number between 1 and $n-1$. Consider that this number is 6 . With this number is make the recombination procedure by adding the first 6 genes of the individual $W_{i}^{t}$ with the last 4 genes of $W_{j}^{t}$; in addition, the first 6 genes of the individual $W_{j}^{t}$ are added to the last 4 genes of $W_{i}^{t}$. The resulting offsprings $\left(\mathrm{Os}_{i}^{t}\right.$ and $\left.\mathrm{Os}_{j}^{t}\right)$ are presented below.

$$
\begin{aligned}
& \mathrm{Os}_{i}^{t}=\left[\begin{array}{lllllllll}
1 & 0 & 1 & 1 & 0 & 1 & 1 & 0 & 1
\end{array}\right], \\
& \mathrm{Os}_{j}^{t}=\left[\begin{array}{lllllllll}
0 & 0 & 1 & 0 & 0 & 0 & 1 & 0 & 0
\end{array}\right] \text {. }
\end{aligned}
$$

\subsubsection{Mutation}

The mutation is a procedure in which a subset of genes is changed. This procedure modifies the initial information of the parents after the recombination procedure. To make this procedure, we use the mutation with a unique point for each offspring, which implies that two random number between 1 and $n-1$ is generated. Suppose that, for $\operatorname{Os}_{i}^{t}$, the random number is 3 and for $\operatorname{Os}_{j}^{t}$ this number is 9 , which produces the following mutations in both offsprings.

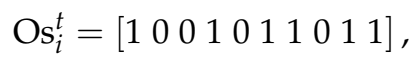

$$
\begin{aligned}
& \mathrm{Os}_{j}^{t}=\left[\begin{array}{llllllllll}
0 & 0 & 1 & 0 & 0 & 0 & 1 & 0 & 1 & 0
\end{array}\right] \text {. }
\end{aligned}
$$

Note that both of the offsprings are different from their parents, since both have new information added by the mutation procedure.

\subsubsection{Fitness Function Evaluations and Replacement of the Population}

Once the offsprings have been created by the selection, recombination, and mutation stages, these are evaluated in the fitness function, i.e., optimization model (14)-(18). Let us suppose that the fitness function of the offspring $\mathrm{Os}_{i}^{t}$ is $z\left(\mathrm{Os}_{i}^{t}\right)$ and the fitness function of the offspring $\mathrm{Os}_{j}^{t}$ is $z\left(\mathrm{Os}_{j}^{t}\right)$. Subsequently, the potential offspring that will be part of the population is selected as the minimum of both fitness function values: (i) select $\mathrm{Os}_{i}^{t}$ if $z\left(\mathrm{Os}_{i}^{t}\right) \geq z\left(\mathrm{Os}_{j}^{t}\right)$, or (ii) select $\mathrm{Os}_{j}^{t}$ otherwise.

In order to decide whether the winner offspring is added to the population, two aspects must be verified: (i) is the fitness function of the winner offspring better than the worst individual in the population? If the answer is positive, then, (ii) is the winner offspring different from each of the individuals in the population? If the answer is also positive, then the winner offspring replace the worst individual in the population.

Note that, if one of the previous questions has a negative response, then a new iteration is started without modification in the population.

\subsubsection{Stopping Criteria}

In order to finalize the searching procedure of the proposed genetic algorithm, the following aspects are considered: 
$\checkmark \quad$ If the maximum number of iteration $t_{\max }$ is reached, the optimization procedure ends by reporting the best solution obtained in the current population.

$\checkmark \quad$ If during $\tau_{\max }$ consecutive iterations, the best objective function has not improved, then the optimization procedure ends by reporting the best solution obtained in the current population.

Remark 5. The genetic algorithm that is presented in the subsections mentioned above is known in the specialized literature as the $C h u$ \& Beasley genetic algorithm [34]. It does not replace all the population individuals in the same iteration, as is made by the classical genetic algorithm.

\subsection{Pseudocode of the Proposed Hybrid Optimization Algorithm}

Algorithm 1 summarized the main aspects of the proposed hybrid GA-SOCP method for optimal placement and sizing of distributed generators in DC distribution networks.

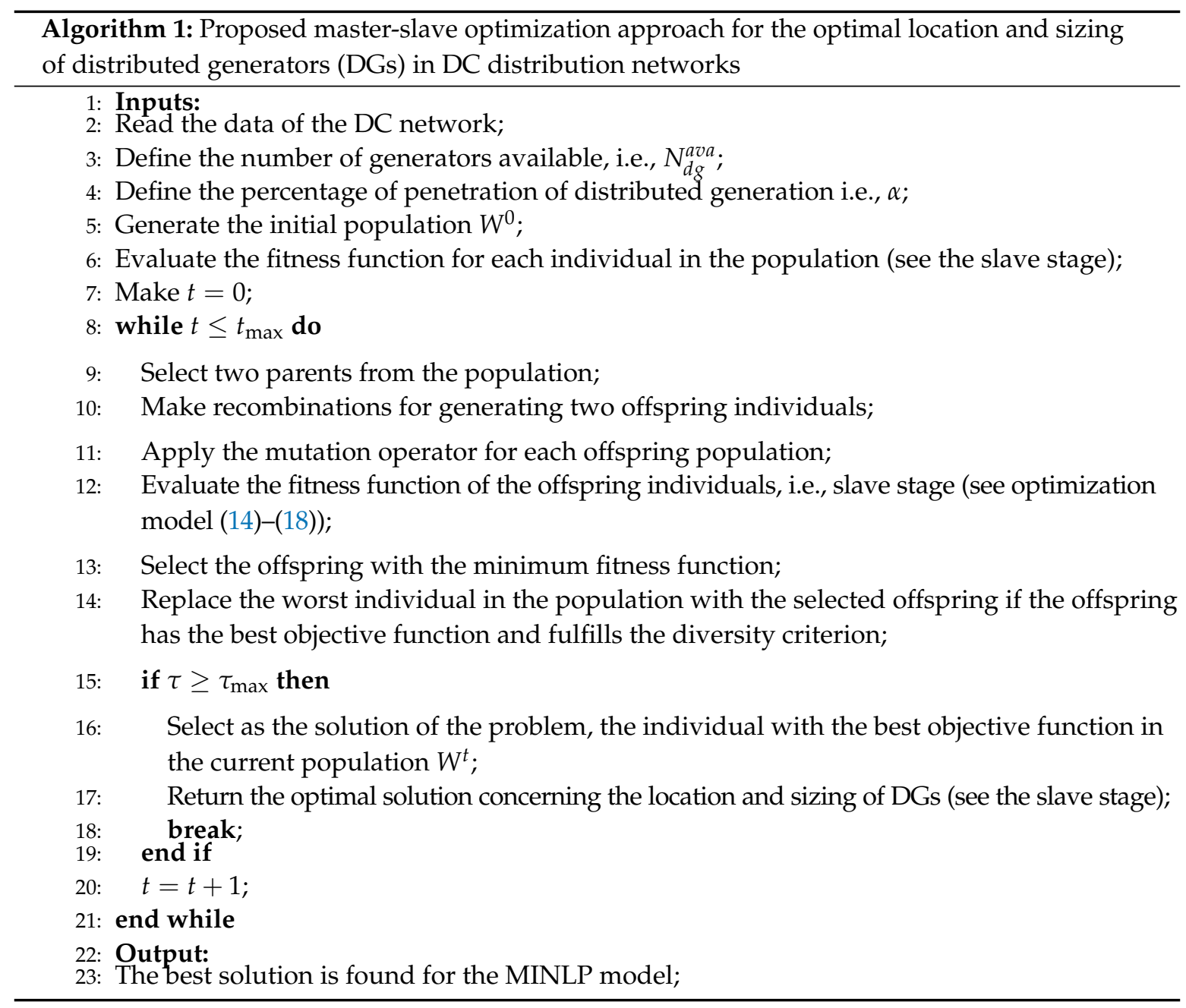

\section{Test Systems}

In this section, the main characteristics of the 21- and 69-node test feeders are presented. These grids are mainly used in power system analysis for DC networks, including voltage stability, power flow analysis, and distributed generators' optimal location [37]. Both of the test feeders are described below. 


\subsection{1-Node Test Feeder}

The 21-node test feeder is a DC distribution network that is composed of 21 nodes and 20 branches that generates a radial configuration where the voltage-controlled source is connected at node 1 . This source supports a voltage profile of $1.00 \mathrm{pu}$ [19]. The complete information regarding constant power consumption and branches are presented in Figure 1 and Table 1, respectively [19].

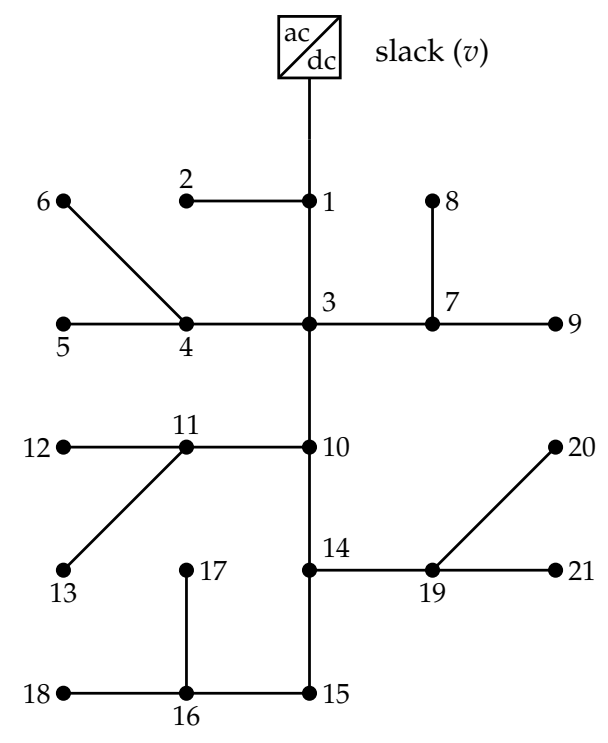

Figure 1. Electrical connection between nodes in the 21-node test feeder.

Table 1. Parametric information of the 21-node test system.

\begin{tabular}{cccccccc}
\hline Node $\boldsymbol{i}$ & Node $\boldsymbol{j}$ & $\boldsymbol{R}_{\boldsymbol{i} \boldsymbol{j}}$ [p.u] & $\boldsymbol{P}_{\boldsymbol{j}}$ [p.u] & Node $\boldsymbol{i}$ & Node $\boldsymbol{j}$ & $\boldsymbol{R}_{\boldsymbol{i} \boldsymbol{j}}$ [p.u] & $\boldsymbol{P}_{\boldsymbol{j}}$ [p.u] \\
\hline 1 & 2 & 0.0053 & 0.70 & 11 & 12 & 0.0079 & 0.68 \\
1 & 3 & 0.0054 & 0.00 & 11 & 13 & 0.0078 & 0.10 \\
3 & 4 & 0.0054 & 0.36 & 10 & 14 & 0.0083 & 0.00 \\
4 & 5 & 0.0063 & 0.04 & 14 & 15 & 0.0065 & 0.22 \\
4 & 6 & 0.0051 & 0.36 & 15 & 16 & 0.0064 & 0.23 \\
3 & 7 & 0.0037 & 0.00 & 16 & 17 & 0.0074 & 0.43 \\
7 & 8 & 0.0079 & 0.32 & 16 & 18 & 0.0081 & 0.34 \\
7 & 9 & 0.0072 & 0.80 & 14 & 19 & 0.0078 & 0.09 \\
3 & 10 & 0.0053 & 0.00 & 19 & 20 & 0.0084 & 0.21 \\
10 & 11 & 0.0038 & 0.45 & 19 & 21 & 0.0082 & 0.21 \\
\hline
\end{tabular}

Note that all of the values in Table 1 are calculated when considering $1 \mathrm{kV}$ and $100 \mathrm{~kW}$ as the voltage and power bases, respectively.

\subsection{9-Node Test Feeder}

This test feeder is a classical AC distribution network that is employed in the literature, such as shunt devices' optimal location for power losses minimization [37]. Notwithstanding, in this study, we used its DC adaptation reported in [37]. Figure 2 depicts the 69-node test feeder configuration and Table 2 reports the branch parameters and load information. Furthermore, for simulation purposes, $12.66 \mathrm{kV}$ and $100 \mathrm{kVA}$ are used as the test system's voltage and power bases. 


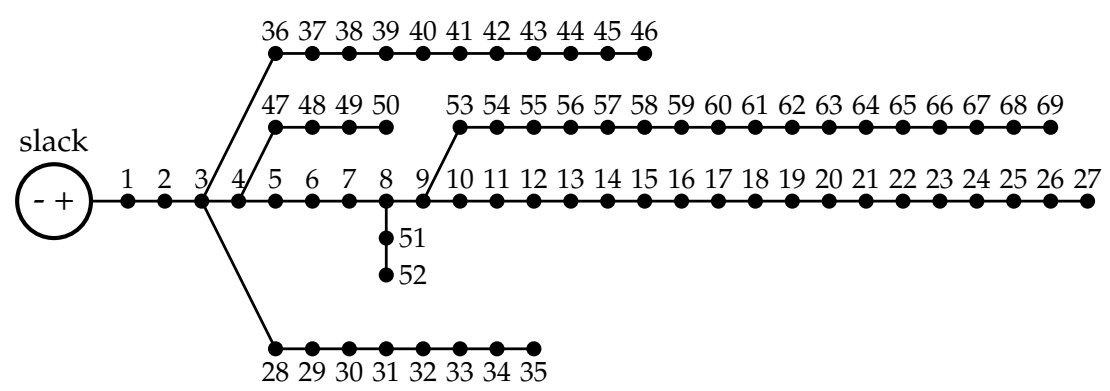

Figure 2. Schematic connection among nodes for the 69-node test feeder.

Table 2. Parametric information of the 69-node test system.

\begin{tabular}{|c|c|c|c|c|c|c|c|}
\hline Node $i$ & Node $j$ & $R_{i j}[\Omega]$ & $P_{j}[\mathrm{~kW}]$ & Node $i$ & Node $j$ & $R_{i j}[\Omega]$ & $P_{j}[\mathrm{~kW}]$ \\
\hline 1 & 2 & 0.0005 & 0 & 3 & 36 & 0.0044 & 26 \\
\hline 2 & 3 & 0.0005 & 0 & 36 & 37 & 0.0640 & 26 \\
\hline 3 & 4 & 0.0015 & 0 & 37 & 38 & 0.1053 & 0 \\
\hline 4 & 5 & 0.0215 & 0 & 38 & 39 & 0.0304 & 24 \\
\hline 5 & 6 & 0.3660 & 2.6 & 39 & 40 & 0.0018 & 24 \\
\hline 6 & 7 & 0.3810 & 40.4 & 40 & 41 & 0.7283 & 102 \\
\hline 7 & 8 & 0.0922 & 75 & 41 & 42 & 0.3100 & 0 \\
\hline 8 & 9 & 0.0493 & 30 & 42 & 43 & 0.0410 & 6 \\
\hline 9 & 10 & 0.8190 & 28 & 43 & 44 & 0.0092 & 0 \\
\hline 10 & 11 & 0.1872 & 145 & 44 & 45 & 0.1089 & 39.22 \\
\hline 11 & 12 & 0.7114 & 145 & 45 & 46 & 0.0009 & 39.22 \\
\hline 12 & 13 & 1.0300 & 8 & 4 & 47 & 0.0034 & 0 \\
\hline 13 & 14 & 1.0440 & 8 & 47 & 48 & 0.0851 & 79 \\
\hline 14 & 15 & 1.0580 & 0 & 48 & 49 & 0.2898 & 384.7 \\
\hline 15 & 16 & 0.1966 & 45 & 49 & 50 & 0.0822 & 384.7 \\
\hline 16 & 17 & 0.3744 & 60 & 8 & 51 & 0.0928 & 40.5 \\
\hline 17 & 18 & 0.0047 & 60 & 51 & 52 & 0.3319 & 3.6 \\
\hline 18 & 19 & 0.3276 & 0 & 9 & 53 & 0.1740 & 4.35 \\
\hline 19 & 20 & 0.2106 & 1 & 53 & 54 & 0.2030 & 26.4 \\
\hline 20 & 21 & 0.3416 & 114 & 54 & 55 & 0.2842 & 24 \\
\hline 21 & 22 & 0.0140 & 5 & 55 & 56 & 0.2813 & 0 \\
\hline 22 & 23 & 0.1591 & 0 & 56 & 57 & 1.5900 & 0 \\
\hline 23 & 24 & 0.3463 & 28 & 57 & 58 & 0.7837 & 0 \\
\hline 24 & 25 & 0.7488 & 0 & 58 & 59 & 0.3042 & 100 \\
\hline 25 & 26 & 0.3089 & 14 & 59 & 60 & 0.3861 & 0 \\
\hline 26 & 27 & 0.1732 & 14 & 60 & 61 & 0.5075 & 1244 \\
\hline 3 & 28 & 0.0044 & 26 & 61 & 62 & 0.0974 & 32 \\
\hline 28 & 29 & 0.0640 & 26 & 62 & 63 & 0.1450 & 0 \\
\hline 29 & 30 & 0.3978 & 0 & 63 & 64 & 0.7105 & 227 \\
\hline 30 & 31 & 0.0702 & 0 & 64 & 65 & 1.0410 & 59 \\
\hline 31 & 32 & 0.3510 & 0 & 65 & 66 & 0.2012 & 18 \\
\hline 32 & 33 & 0.8390 & 10 & 66 & 67 & 0.0047 & 18 \\
\hline 33 & 34 & 1.7080 & 14 & 67 & 68 & 0.7394 & 28 \\
\hline 34 & 35 & 1.4740 & 4 & 68 & 69 & 0.0047 & 28 \\
\hline
\end{tabular}

\section{Computational Implementation}

The proposed hybrid optimization model, i.e., the GA-SOCP method, has been implemented in the MATLAB software version $2019 b$ on a desktop computer with an INTEL(R) Core(TM) i7-7700 2.8-GHz processor and 16.0 GB of RAM running on a 64-bit version of Microsoft Windows 10 Home. To validate our proposed MI-SOCP formulation solved with the proposed hybrid GA-SOCP approach, we employed different MINLP solvers that were available in the GAMS software (i.e., SBB and CONOPT solvers), and some metaheuristic approaches reported in [37]. In [37], the genetic algorithm has been combined with three continuous methods, as follows: black-hole optimizer (GA-BHO), 
particle swarm optimization (GA-PSO), and continuous genetic algorithm (GA-CGA). In addition, the following simulating conditions are considered:

$\checkmark \quad$ Three distributed generators are available for installation in the DC test feeder; wherein, the case of the 21-node test system, their maximum individual allowed size is $1.5 \mathrm{pu}$ and, in the case of the 69-node test feeder, this bound is $12 \mathrm{pu}$.

$\checkmark \quad$ The maximum power penetration in the 21-node test feeder is $40 \%$ and, in the case of the 69 -node test feeder, the maximum penetration is $40 \%$ regarding the total power demand of each one of the DC networks.

Remark 6. All the genetic algorithms employed as the master stage in the proposed and comparison methods have adjusted using a trial and error procedure, which have been produced as parameters: 10 individuals in the population, 100 iterations, recombination, and mutation rates $50 \%$. In addition, to evaluate statistically speaking these methods, 100 consecutive iterations were made for each method.

\subsection{1-Node Test Feeder}

Table 3 reports the numerical comparisons between the proposed hybrid optimization method and the literature approaches for the 21-node test feeder. Note that this problem's optimal solution corresponds to nodes 9,12 , and 16, with power injections of $0.8350 \mathrm{pu}, 1.0258 \mathrm{pu}$, and $1.4632 \mathrm{pu}$, respectively.

Table 3. Comparison of different approaches in the 21-node test feeder.

\begin{tabular}{cccccc}
\hline Method & Nodes & $\sigma$ [p.u.] & $\mu$ [p.u.] & $\min [p . u]$. & $t_{\text {ave }}$ [s] \\
\hline GA-BHO & $\{9,12,16\}$ & $2.2761 \times 10^{-03}$ & 0.0368 & 0.0318 & 111.9440 \\
GA-CGA & $\{9,12,16\}$ & $1.3537 \times 10^{-03}$ & 0.0329 & 0.0311 & 33.1974 \\
GA-PSO & $\{9,12,16\}$ & $1.8437 \times 10^{-03}$ & 0.0319 & 0.0306 & 58.0934 \\
MIQP & $\{9,12,16\}$ & --- & 0.0306 & 0.0306 & 8.9688 \\
SSB & $\{9,12,16\}$ & --- & 0.0306 & 0.0306 & 5.3653 \\
GA-SOCP & $\{9,12,16\}$ & $1.1050 \times 10^{-03}$ & 0.0309 & 0.0306 & 52.146 \\
\hline
\end{tabular}

From Table 3, we can observe that:

$\checkmark \quad$ All of the comparison methods, as well as the proposed approach, identifies the best optimal location in nodes 9,12 , and 16 . However, due to the aleatory nature of the continuous metaheuristics (i.e., BHO, PSO, and CGA), both of them are stuck local optimums, and only the GA-PSO approach can deal with the global optimal solution.

$\checkmark \quad$ The mixed-integer quadratic programming (MIQP) model and the SBB solver (for the exact MINLP model) reach the global optimal solution with the lowest computational effort, which is attributable to the efficiency of the GAMS interface.

$\checkmark \quad$ The standard deviation of the proposed GA-SOCP programming is the lowest when compared with metaheuristics; this situation occurs because the proposed approach works with an exact optimization method based on second-order cone programming that has a unique solution for each combination of DGs provided by the GA in the master stage.

Figure 3 presents the histogram after the 100 consecutive evaluations of the proposed methodology for optimal placement and sizing distributed generators in DC distribution networks in order to present the efficiency of the proposed GA-SOCP approach. 


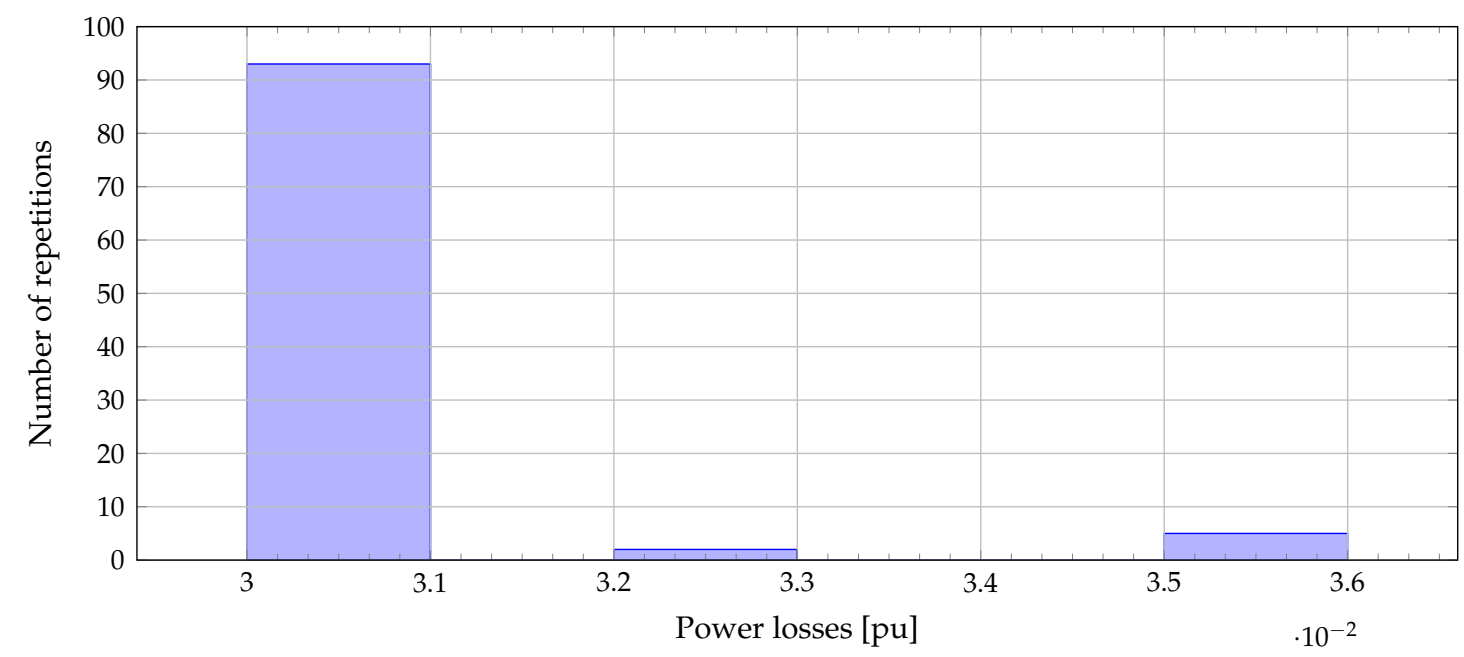

Figure 3. Behavior of the proposed hybrid GA-PSO after 100 consecutive evaluations in the 21-node test feeder.

Note that the histogram presented in Figure 3 shows that more than 90 solutions (93 exactly) reached by the proposed hybrid GA-SOCP method has the capability of finding the global optimal solution (i.e., $0.0306 \mathrm{pu}$ ) regarding power losses, which confirms the efficiency and robustness of the studied methodology for optimal placement and sizing DGs in DC distribution networks.

Remark 7. We can ensure that the solution finds by the proposed GA-SOCP approach is the optimal one, since all the possibilities (i.e., 1140 options) for the location of DGs in the 21-node test feeder have been evaluated comprehensively via nested loops, which have confirmed that nodes 9, 12 and 16 are the best possible location for these DGs reaching a final power losses of $0.0306 \mathrm{pu}$. It is worth mentioning that this exhaustive evaluation takes about $800 \mathrm{~s}$.

Figure 4, which presents the percentage of improvement reached by the comparative methods in contrast to the proposed hybrid optimization approach, shows the total grid improvement about the power losses minimization.

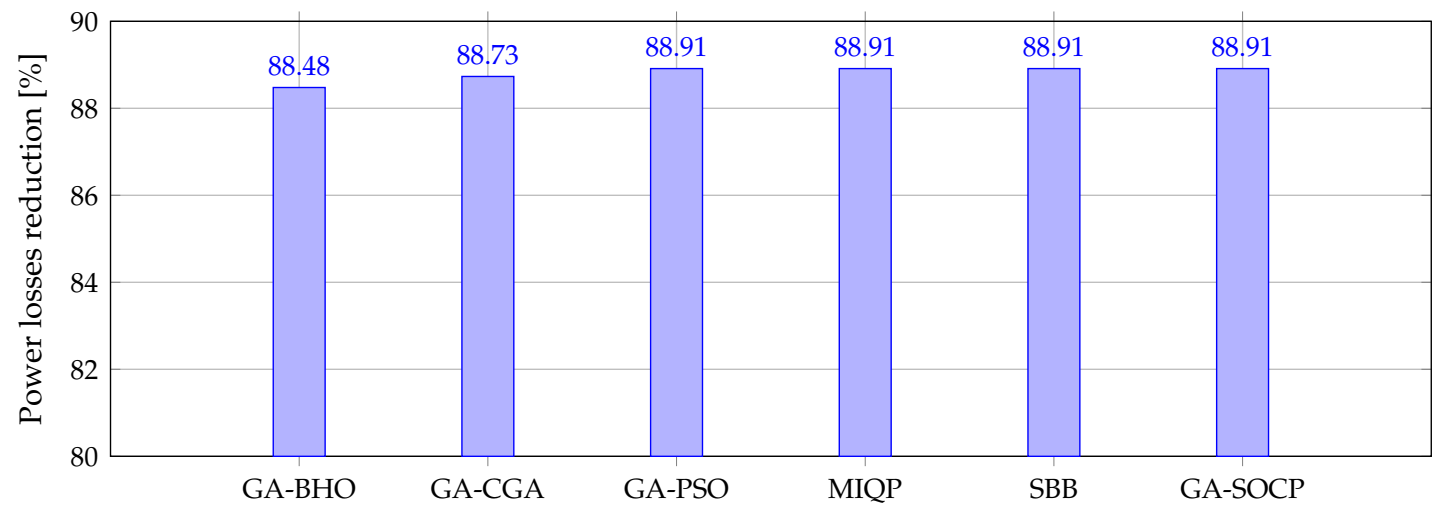

Figure 4. Percentage of improvement regarding power losses minimization in the 21-node test feeder.

Figure 4 shows the positive impact that has the inclusion of distributed generation in DC distribution networks, since all of the comparative methods and the proposed hybrid approach present improvements that are higher than $88 \%$ with a total penetration of $60 \%$ in a distributed generation regarding total power consumption. In addition, these results confirm the global capabilities of the proposed hybrid GA-SOCP method for dealing with the problem of the optimal location and sizing DGs in DC feeders. 


\subsection{9-Node Test Feeder}

Table 4 presents the numerical results of the proposed and comparative methods. These results allow observing that:

$\checkmark \quad$ All of the comparative methods are stuck in locally optimal solutions, including the MIQP approach and the MINLP model solved in GAMS through the SBB solver since these finds final power losses about $0.1660 \mathrm{pu}$ and $0.1574 \mathrm{pu}$, respectively. However, the best optimal solution is reached by the GA-SOCP method with $0.1573 \mathrm{pu}$.

$\checkmark \quad$ Some of the metaheuristic approaches (i.e., GA-CGA and GA-PSO) find the best positions for installing the GDs being these nodes 21, 61, and 64; nevertheless, due to the random procedures in the slave stages, these find local solutions.

$\checkmark$ Even if standard deviations of the GA-PSO and GA-CGA are lower than the proposed GA-SOCP, these values imply that these methods are always trapped in the local solutions, and they do not have the capacity of scape from these solutions to explore other promissory regions of the solution space.

Table 4. Comparison of different approaches in the 69-node test feeder.

\begin{tabular}{cccccc}
\hline Method & Nodes & $\sigma$ [p.u.] & $\mu$ [p.u.] & $\min [p . u]$. & $t_{\text {ave }}[\mathrm{s}]$ \\
\hline GA-BHO & $\{23,6167\}$ & $2.5207 \times 10^{-03}$ & 0.1633 & 0.1593 & 713.7193 \\
GA-CGA & $\{21,61,64\}$ & $3.4801 \times 10^{-04}$ & 0.1648 & 0.1603 & 218.0169 \\
GA-PSO & $\{21,61,64\}$ & $5.4023 \times 10^{-04}$ & 0.1689 & 0.1588 & 378.4731 \\
MIQP & $\{22,61,64\}$ & --- & 0.1660 & 0.1660 & 24.1868 \\
SBB & $\{22,61,65\}$ & --- & 0.1574 & 0.1574 & 12.4270 \\
GA-SOCP & $\{21,61,64\}$ & $3.1295 \times 10^{-03}$ & 0.1591 & 0.1573 & 66.9100 \\
\hline
\end{tabular}

Note that the best optimal solution reported by the GA-SOCP proposed approach connects DGs in nodes 21, 61, and 64 with generation capabilities of about $1.4140 \mathrm{pu}, 10.2630 \mathrm{pu}$, and 3.8803 , respectively. Once again, we can confirm that this solution is the global optimum, since, after the exhaustive evaluation of the solution space (i.e., 50116 possible locations) via nested loops, the same set of nodes and power generations have been identified as the best global solution. It is important to highlight that this exhaustive evaluation takes about $7000 \mathrm{~s}$ to revise the whole solution space, which implies that $66.91 \mathrm{~s}$ reported by the proposed GA-SOCP approach demonstrates its efficiency and robustness global finding capabilities.

Figure 5 presents the histogram with the optimization behavior of the proposed GA-SOCP method for locating and sizing DGs in DC distribution networks.

From Figure 5, it can be noted that more than $70 \%$ of the solutions that were found by the proposed GA-SOCP method find the global optimal solution, and $24 \%$ of the additional solutions are better than the mean solutions reported in Table 4 (see $\mu$ column). These results confirm the proposed GA-SOCP optimization approach's efficiency and robustness for solving the optimal placement and sizing of DGs in DC distribution networks with global optimum finding capabilities.

Figure 6 presents the percentage of power losses improvement reached by the comparative and the proposed optimization approaches.

Note that the percentage of reduction of power losses reported in plot 6 confirms the global optimization capabilities of the proposed GA-SOCP approach, since it is the only one methodology that reports the highest percentage of reduction with $89.78 \%$, only followed by the SBB methodology with $89.77 \%$ of reduction. 


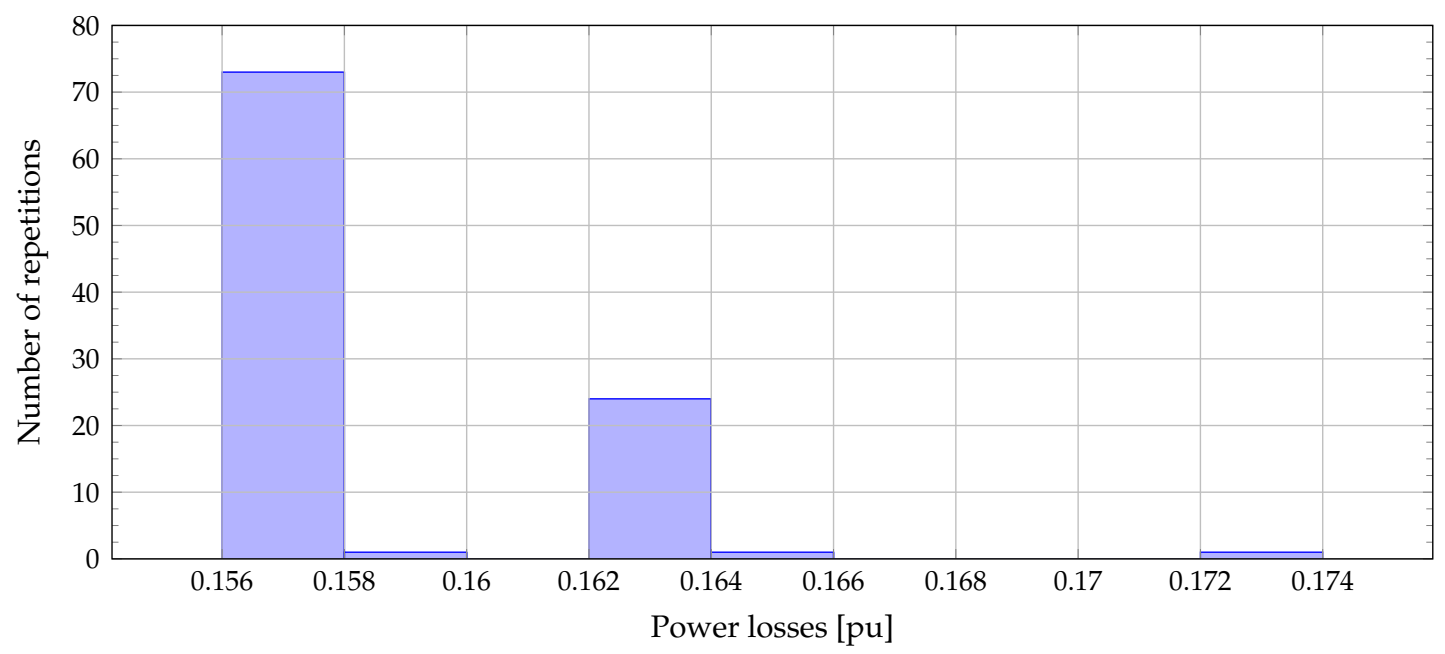

Figure 5. Behavior of the proposed hybrid GA-PSO after 100 consecutive evaluations in the 69-node test feeder.

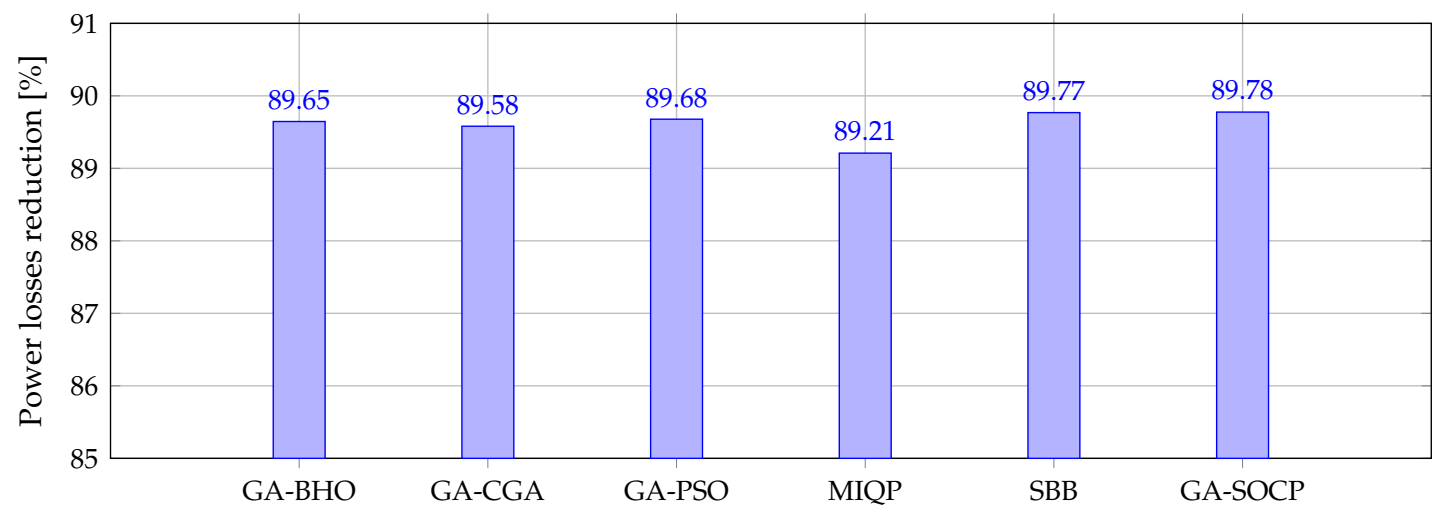

Figure 6. Percentage of improvement regarding power losses minimization in the 69-node test feeder.

\subsection{General Commentaries}

After applying the comparative and the proposed optimization methodology for optimal locating and sizing DGs in DC distribution networks, we can affirm that:

$\checkmark \quad$ The processing times in both test systems have been lower than $70 \mathrm{~s}$, which implies that for planning purposes, our approach is perfectly applicable since, with a fraction of the time taken by the exhaustive search method, this can deal with the global optimal solution. In addition, the processing times of the comparative metaheuristic methods increase rapidly as a function of the test feeder size (see the last columns of Tables 3 and 4) with the main disadvantage that due to the random behavior of the slave stages, i.e., BHO, CGA, and PSO, it is not possible to find the optimal solution of the studied optimization problem.

$\checkmark \quad$ The methodologies implemented in GAMS, i.e., the MIQP and the MINLP model, both solved with the SBB tool available in GAMS, have speedy processing times. However, the linearization of the power flow equations in the MIQP approach and the nonlinear and non-convex structure of the exact MINLP model make that these get stuck in optimal locations as the solution space grows.

$\checkmark \quad$ The main advantages of the proposed hybrid GA-SOCP master-slave optimization method are: (i) the possibility of using a compact codification with integer numbers (nodes) that allows for guaranteeing feasibility in all the stages of the genetic algorithm procedures, and (ii) the guarantee of the global optimal solution for each combination of distributed generators thanks to the second-order cone structure of the optimal power flow problem in the slave stage. 


\section{Conclusions and Future Works}

A hybrid master-slave optimization approach for optimal placement and sizing distributed generators in DC distribution networks while using a combination of the classical Chu \& Beasley genetic algorithm in the master stage and a second-order cone programming model in the slave stage, i.e., GA-SOCP. This algorithm has an important advantage regarding classical metaheuristics, since solving the optimal power flow problem for optimal dimensioning the distributed generators provided by the master stage with a SCOP model guarantees the existence and uniqueness of the optimal solution, which is not possible with combination methods, such as BHO, CGA, and PSO, where random procedures affect the optimization process. Numerical comparisons with classical metaheuristics and exact methodologies demonstrate that, for the studied test feeders, the proposed GA-SOCP approach deals with the global optimal solution. Simultaneously, it is not the case of the comparative methods in the 69-node test feeder scenario. The effectiveness of the proposed GA-SOCP method to find the optimal solution after 100 consecutive evaluations is about $73 \%$ for the 21-node test feeder, and $93 \%$ in the case of the 69-node test system, which implies the high efficiency of the studied hybrid optimization approach to deal with MINLP problems.

Regarding future works, it will be possible to make the following researches: (i) extend the proposed optimization methodology to the optimal location and sizing of distributed generators and capacitor banks in AC distribution networks, (ii) to apply the proposed GA-SOCP approach to the problem of the optimal placement and sizing battery energy storage systems in AC and DC networks, and (iii) to develop an economic dispatch approach to optimal locating renewable generation at isolated distribution networks when considering variations in the load curve and the primary energy resources.

Author Contributions: Conceptualization, O.D.M. and W.G.-G.; Methodology, O.D.M. and W.G.-G.; Investigation, O.D.M., W.G.-G., and L.F.G.-N.; Writing-review and editing, O.D.M., W.G.-G., and L.F.G.-N. All authors have read and agreed to the published version of the manuscript.

Funding: This research received no external funding.

Acknowledgments: This work was partially supported by the National Scholarship Program Doctorates of the Administrative Department of Science, Technology, and Innovation of Colombia (COLCIENCIAS), by calling contest 727-2015.

Conflicts of Interest: The authors declare no conflicts of interest.

\section{Abbreviations}

The following abbreviations are used in this manuscript:

$\begin{array}{ll}\text { Acronyms } & \\ \text { AC } & \text { Alternating Current } \\ \text { DG } & \text { Distributed Generator } \\ \text { GA } & \text { Chu \& Beasley genetic algorithm } \\ \text { HSM } & \text { Hyperplanes search method } \\ \text { NLP } & \text { Nonlinear Programming } \\ \text { CVX } & \text { Matlab Software for Disciplined Convex Programming } \\ \text { GAMS } & \text { General Algebraic Modeling System } \\ \text { MI } & \text { Mixed-integer } \\ \text { MINLP } & \text { Mixed-integer nonlinear programming } \\ \text { SOCP } & \text { Second-order cone programming } \\ \text { SDP } & \text { Semidefinite programming }\end{array}$




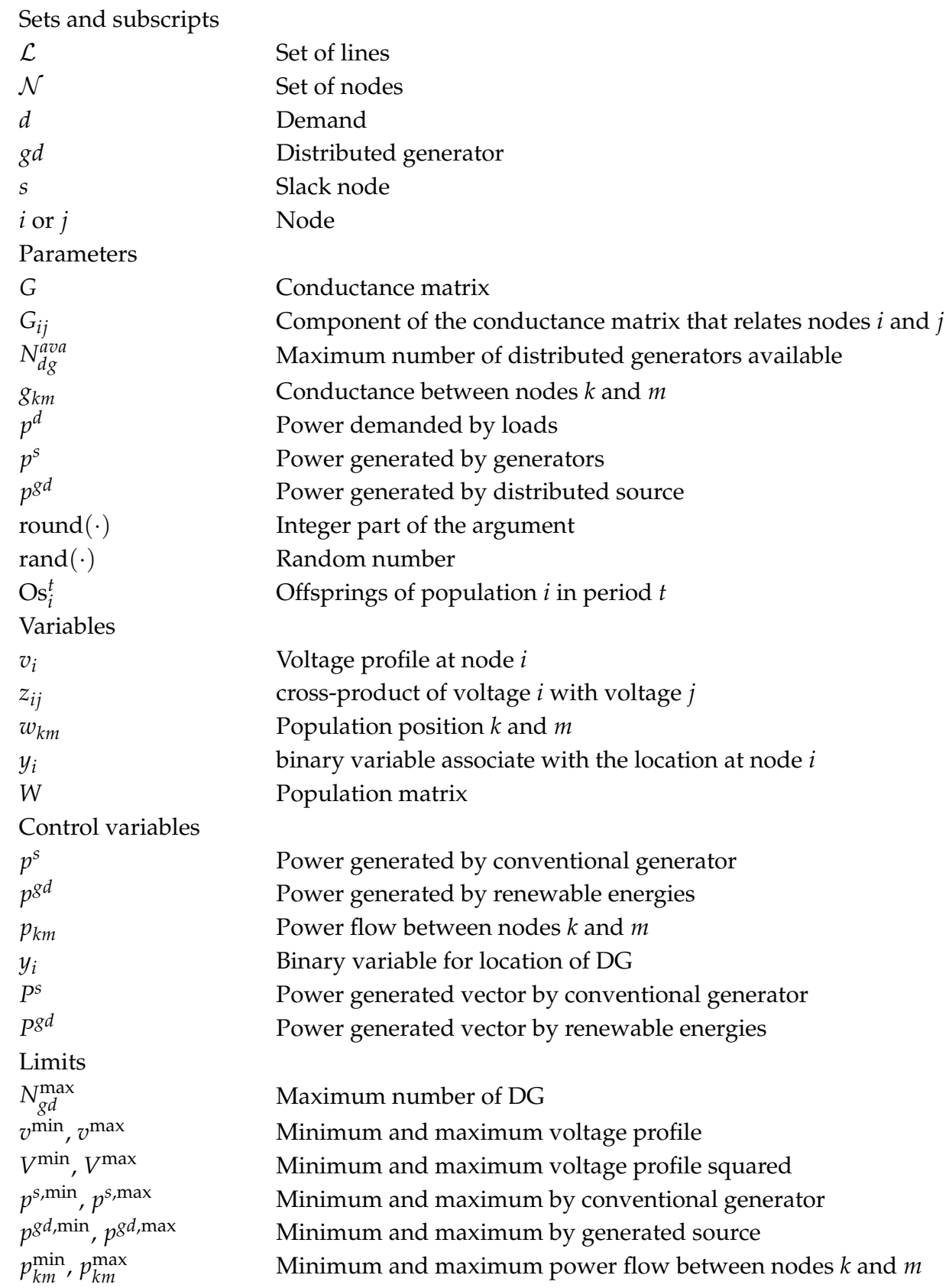

\section{References}

1. Kwon, M.; Choi, S. Control scheme for autonomous and smooth mode switching of bidirectional DC-DC converters in a DC microgrid. IEEE Trans. Power Electron. 2017, 33, 7094-7104. [CrossRef]

2. Garcés, A. Stability Analysis of DC-Microgrids: A Gradient Formulation. J. Control Autom. Electr. Syst. 2019, 30, 985-993. [CrossRef]

3. Wang, L.; Wang, K.H.; Lee, W.J.; Chen, Z. Power-flow control and stability enhancement of four parallel-operated offshore wind farms using a line-commutated HVDC link. In Wind Energy Conversion Systems; Springer: Berlin/Heidelberg, Germany, 2012; pp. 385-414.

4. Gavriluta, C.; Candela, I.; Citro, C.; Luna, A.; Rodriguez, P. Design considerations for primary control in multi-terminal VSC-HVDC grids. Electr. Power Syst. Res. 2015, 122, 33-41. [CrossRef]

5. Simiyu, P.; Xin, A.; Wang, K.; Adwek, G.; Salman, S. Multiterminal Medium Voltage DC Distribution Network Hierarchical Control. Electronics 2020, 9, 506. [CrossRef]

6. Justo, J.J.; Mwasilu, F.; Lee, J.; Jung, J.W. AC-microgrids versus DC-microgrids with distributed energy resources: A review. Renew. Sustain. Energy Rev. 2013, 24, 387-405. [CrossRef] 
7. Parhizi, S.; Lotfi, H.; Khodaei, A.; Bahramirad, S. State of the Art in Research on Microgrids: A Review. IEEE Access 2015, 3, 890-925. [CrossRef]

8. Kumar, D.; Zare, F.; Ghosh, A. DC microgrid technology: system architectures, AC grid interfaces, grounding schemes, power quality, communication networks, applications, and standardizations aspects. IEEE Access 2017, 5, 12230-12256. [CrossRef]

9. Lotfi, H.; Khodaei, A. AC versus DC microgrid planning. IEEE Trans. Smart Grid 2015, 8, 296-304. [CrossRef]

10. Gelani, H.; Dastgeer, F.; Siraj, K.; Nasir, M.; Niazi, K.; Yang, Y. Efficiency Comparison of AC and DC Distribution Networks for Modern Residential Localities. Appl. Sci. 2019, 9, 582. [CrossRef]

11. Rouzbehi, K.; Miranian, A.; Candela, J.I.; Luna, A.; Rodriguez, P. A Generalized Voltage Droop Strategy for Control of Multiterminal DC Grids. IEEE Trans. Ind. Appl. 2015, 51, 607-618. [CrossRef]

12. Rouzbehi, K.; Miranian, A.; Luna, A.; Rodriguez, P. DC Voltage Control and Power Sharing in Multiterminal DC Grids Based on Optimal DC Power Flow and Voltage-Droop Strategy. IEEE J. Emerg. Sel. Top. Power Electron. 2014, 2, 1171-1180. [CrossRef]

13. Garcés, A. Convex Optimization for the Optimal Power Flow on DC Distribution Systems. In Handbook of Optimization in Electric Power Distribution Systems; Springer: Berlin/Heidelberg, Germany, 2020; pp. 121-137.

14. Garcés, A. On the Convergence of Newton's Method in Power Flow Studies for DC Microgrids. IEEE Trans. Power Syst. 2018, 33, 5770-5777. [CrossRef]

15. Garces, A. Uniqueness of the power flow solutions in low voltage direct current grids. Electr. Power Syst. Res. 2017, 151, 149-153. [CrossRef]

16. Hamad, A.A.; El-Saadany, E.F. Multi-agent supervisory control for optimal economic dispatch in DC microgrids. Sustain. Cities Soc. 2016, 27, 129-136. [CrossRef]

17. Barabanov, N.; Ortega, R.; Griñó, R.; Polyak, B. On Existence and Stability of Equilibria of Linear Time-Invariant Systems With Constant Power Loads. IEEE Trans. Circuits Syst. I 2016, 63, 114-121. [CrossRef]

18. Chauhan, R.K.; Chauhan, K.; Guerrero, J.M. Controller design and stability analysis of grid connected DC microgrid. J. Renew. Sustain. Energy 2018, 10, 035101. [CrossRef]

19. Montoya, O.D.; Gil-González, W.; Grisales-Noreña, L. Relaxed convex model for optimal location and sizing of DGs in DC grids using sequential quadratic programming and random hyperplane approaches. Int. J. Electr. Power Energy Syst. 2020, 115, 105442. [CrossRef]

20. Grisales-Noreña, L.; Montoya, O.D.; Ramos-Paja, C.A. An energy management system for optimal operation of BSS in DC distributed generation environments based on a parallel PSO algorithm. J. Energy Storage 2020, 29, 101488. [CrossRef]

21. Fantauzzi, M.; Lauria, D.; Mottola, F.; Scalfati, A. Sizing energy storage systems in DC networks: A general methodology based upon power losses minimization. Appl. Energy 2017, 187, 862-872. [CrossRef]

22. Castillo-Calzadilla, T.; Macarulla, A.M.; Kamara-Esteban, O.; Borges, C.E. A case study comparison between photovoltaic and fossil generation based on direct current hybrid microgrids to power a service building. J. Clean. Prod. 2020, 244, 118870. [CrossRef]

23. Chiodo, E.; Fantauzzi, M.; Lauria, D.; Mottola, F. A Probabilistic Approach for the Optimal Sizing of Storage Devices to Increase the Penetration of Plug-in Electric Vehicles in Direct Current Networks. Energies 2018, 11, 1238. [CrossRef]

24. Montoya, O.D. A convex OPF approximation for selecting the best candidate nodes for optimal location of power sources on DC resistive networks. Eng. Sci. Technol. Int. J. 2020, 23, 527-533. [CrossRef]

25. Montoya, O.D.; Garrido, V.M.; Grisales-Noreña, L.F.; Gil-González, W.; Garces, A.; Ramos-Paja, C.A. Optimal Location of DGs in DC Power Grids Using a MINLP Model Implemented in GAMS. In Proceedings of the 2018 IEEE 9th Power, Instrumentation and Measurement Meeting (EPIM), Salto, Uruguay, 14-16 November 2018; pp. 1-5.

26. Montoya, O.D.; Grisales-Noreña, L.F.; Gil-González, W.; Alcalá, G.; Hernandez-Escobedo, Q. Optimal Location and Sizing of PV Sources in DC Networks for Minimizing Greenhouse Emissions in Diesel Generators. Symmetry 2020, 12, 322. [CrossRef]

27. Grisales-Noreña, L.F.; Garzon-Rivera, O.D.; Montoya, O.D.; Ramos-Paja, C.A. Hybrid Metaheuristic Optimization Methods for Optimal Location and Sizing DGs in DC Networks. In Proceedings of the Workshop on Engineering Applications, Santa Marta, Colombia, 16-18 October 2019; Springer: Berlin/Heidelberg, Germany, 2019; pp. 214-225. 
28. Huang, L.; Chen, Z.; Cui, Q.; Zhang, J.; Wang, H.; Shu, J. Optimal planning of renewable energy source and energy storage in a medium- and low-voltage distributed AC/DC system in China. J. Eng. 2019, 2019, 2354-2361. [CrossRef]

29. Li, J.; Liu, F.; Wang, Z.; Low, S.H.; Mei, S. Optimal Power Flow in Stand-Alone DC Microgrids. IEEE Trans. Power Syst. 2018, 33, 5496-5506. [CrossRef]

30. Benson, H.Y.; Sağlam, Ü. Mixed-Integer Second-Order Cone Programming: A Survey. In Theory Driven by Influential Applications; INFORMS: Catonsville, MD, USA, 2013; pp. 13-36.

31. Li, Q.; Vittal, V. Convex Hull of the Quadratic Branch AC Power Flow Equations and Its Application in Radial Distribution Networks. IEEE Trans. Power Syst. 2018, 33, 839-850. [CrossRef]

32. Farivar, M.; Low, S.H. Branch Flow Model: Relaxations and Convexification-Part I. IEEE Trans. Power Syst. 2013, 28, 2554-2564. [CrossRef]

33. Lambora, A.; Gupta, K.; Chopra, K. Genetic Algorithm- A Literature Review. In Proceedings of the 2019 International Conference on Machine Learning, Big Data, Cloud and Parallel Computing (COMITCon), Faridabad, India, 14-16 February 2019; IEEE: Piscataway, NJ, USA, 2019. [CrossRef]

34. Montoya, O.D.; Gil-González, W.; Orozco-Henao, C. Vortex search and Chu-Beasley genetic algorithms for optimal location and sizing of distributed generators in distribution networks: A novel hybrid approach. Eng. Sci. Technol. Int. J. 2020. [CrossRef]

35. Sahin, O.; Akay, B. Comparisons of metaheuristic algorithms and fitness functions on software test data generation. Appl. Soft Comput. 2016, 49, 1202-1214. [CrossRef]

36. Badem, H.; Basturk, A.; Caliskan, A.; Yuksel, M.E. A new hybrid optimization method combining artificial bee colony and limited-memory BFGS algorithms for efficient numerical optimization. Appl. Soft Comput. 2018, 70, 826-844. [CrossRef]

37. Montoya, O.D.; Gil-González, W. A MIQP model for optimal location and sizing of dispatchable DGs in DC networks. Energy Syst. 2020, 1-22. [CrossRef]

Publisher's Note: MDPI stays neutral with regard to jurisdictional claims in published maps and institutional affiliations.

(C) 2020 by the authors. Licensee MDPI, Basel, Switzerland. This article is an open access article distributed under the terms and conditions of the Creative Commons Attribution (CC BY) license (http://creativecommons.org/licenses/by/4.0/). 